\title{
Denominator estimation: approaches in the Hamburg paediatric sentinel network
}

\author{
Marco Kellerhof, Klaus Gritz, Helmut Brand
}

\begin{abstract}
Study objective - The aims were to develop an estimator for the size of paediatric practices to be used as a denominator for purposes of comparison; to analyse the age structure of the patients attending paediatric practices and to check the necessity for an age specific denominator; and to validate the denominator information by other available data.

Design - This was an observational study. Settinglparticipants - A sentinel network was set up comprising 26 self selected paediatric practices. Weekly patient contacts in relation to age and sex were counted three times during the study period of two years. In addition, accounting data, including the total number of children treated in a given three month period (quarter), were available.

Main results - Weekly patient contact counts were stable over time, not in terms of the absolute number of contacts but in the rank positions of the practices $\left(r_{s}=\right.$ $0 \cdot 86$ ) and in their age structure. The age distribution of weekly patient contacts differed significantly between the practices. Cross validation of the weekly contact count by means of the quarterly accounting data resulted in a rank correlation of $r_{s}=0.90$.

Conclusions - Sentinel networks with paediatric practices should use age specific denominator information. Weekly contact group, estimated by counts in a sample of weeks, is a stable and easily available denominator for sentinel practices in the context of the German health care system.
\end{abstract}

Behoerde für Arbeit, Gesundheit und Soziales, Tesdorpfstr 8, D-20148 Hamburg, Germany M Kellerhof

Berufsverband der Kinderaerzte Deutschlands, Landesverband, Hamburg, Germany

K Gritz

Behoerde für Arbeit, Gesundheit und

Soziales,

Hamburg,

Germany

H Brand

Correspondence to: M Kellerhof.
(FEpidemiolCommunity Health 1995;49(Suppl 1):26-28)

Community health analyses in Germany tend to be restricted to mortality and demographic indicators, sometimes completed by survey data. Much is still unknown about health problems that entail ambulatory care. In Hamburg, the Department of Labour, Health and Social Affairs and the Hamburg Association of Paediatricians took the initiative to monitor health problems in children. After a successful pilot catalogue of items to be monitored over a two year period (May 1992 to April 1994). Doctors marked on a weekly checklist every patient who had expiratory wheezing, atopic dermatitis, indoor and outdoor accidental injuries, or suspected child abuse (emotional, physical or project, ${ }^{1-3}$ intensive discussions resulted in a sexual). Additional information included the sex and age (in four age groups) of the patient. Thirty eight paediatricians in 26 practices participated voluntarily in the sentinel network. They represent more than a quarter of all paediatric practices and one third of all paediatricians undertaking outpatient care in Hamburg.

The aim of the Hamburg paediatric sentinel project is to detect and study local "epidemics" of health problems in ambulatory care and their correlates. ${ }^{4}$ The main method used to achieve this is week to week comparison of all of the practices or regional subgroups of practices.

As in other sentinel projects in countries where doctors do not have fixed patient lists, no information was available about the size and age structure of the population served by each paediatrician. In Germany, parents are free to choose a paediatric (or general) practice for the medical treatment of their children, wherever this practice is located. Most of the parents will choose a doctor nearby, but no clear cut catchment areas exist for the practices. For this reason, practice denominators can not be derived from population census data. And the fact that the proportion of children treated by general practitioners (and not by paediatric practitioners) may vary from area to area makes this even more difficult.

For the intended time series analyses, practice specific denominator information was indispensable since almost every week one or more practices dropped out for reasons of holiday, illness etc. Knowledge of the size and structure of these practices was necessary to enable comparisons to be made.

The objectives of the present study were as follows:

- To develop an estimator for the size of a paediatric practices;

- To analyse the age structure of the patients and to decide whether an age specific denominator is necessary;

- To validate the denominator information by other available data and to check the possibility of estimating missing practice denominator data.

\section{Methods}

In addition to the health problems checklist, paediatricians counted every patient consultation within one week in October 1992. This week was selected for two reasons: firstly, the doctors had to become familiar with the health problems checklist in May and June and would not accept a parallel census; secondly, 
there was no week between July and September in which no practice dropped out because of summer holidays.

Denominator counting was repeated twice in the study period (in March 1993 and in March 1994; data for March 1994 are not included in this paper) to validate the structure of the weekly contact group. Patient contacts were counted in the same age and sex groups as the nominator. A continuous or even monthly census of all patient contacts was impossible, because long term sentinel networks can only be run if the workload for the participating doctors is restricted to the minimum possible.

Doctors were further asked to make available their quarterly accounting data. These data include the number of treated children insured under the Federal Health Protection Act. For every child counted there is at least one doctorpatient contact in the given quarter, but the number of total contacts with this child is unknown. Leaving aside the (small) number

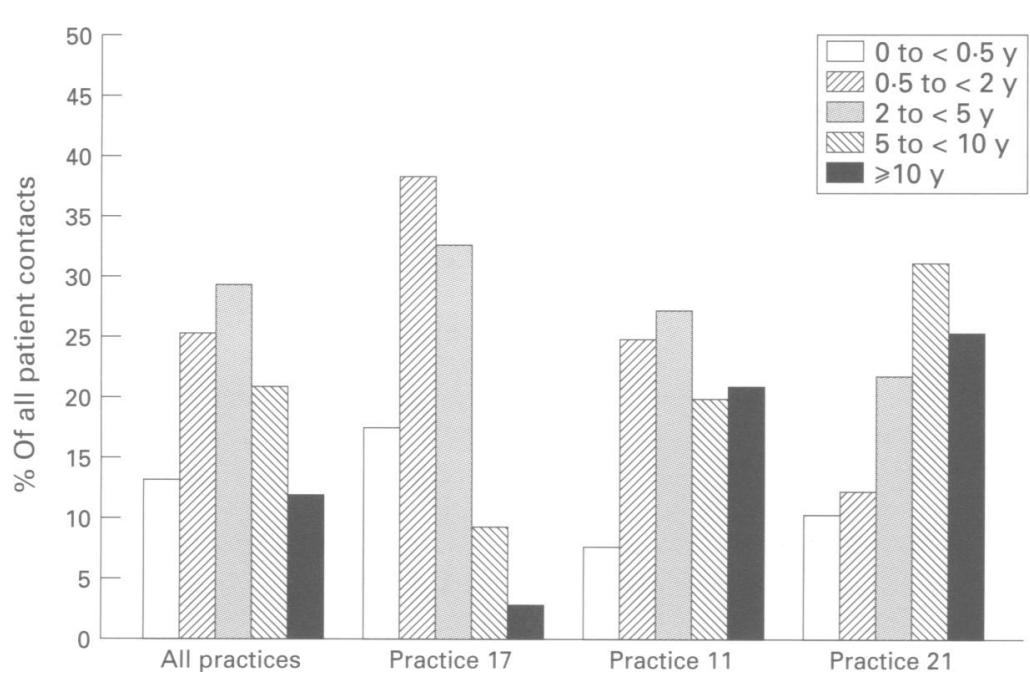

Figure 1 Age distribution of children in all the sentinel network practices combined and in three practices $(17,11$, and 21$)$.

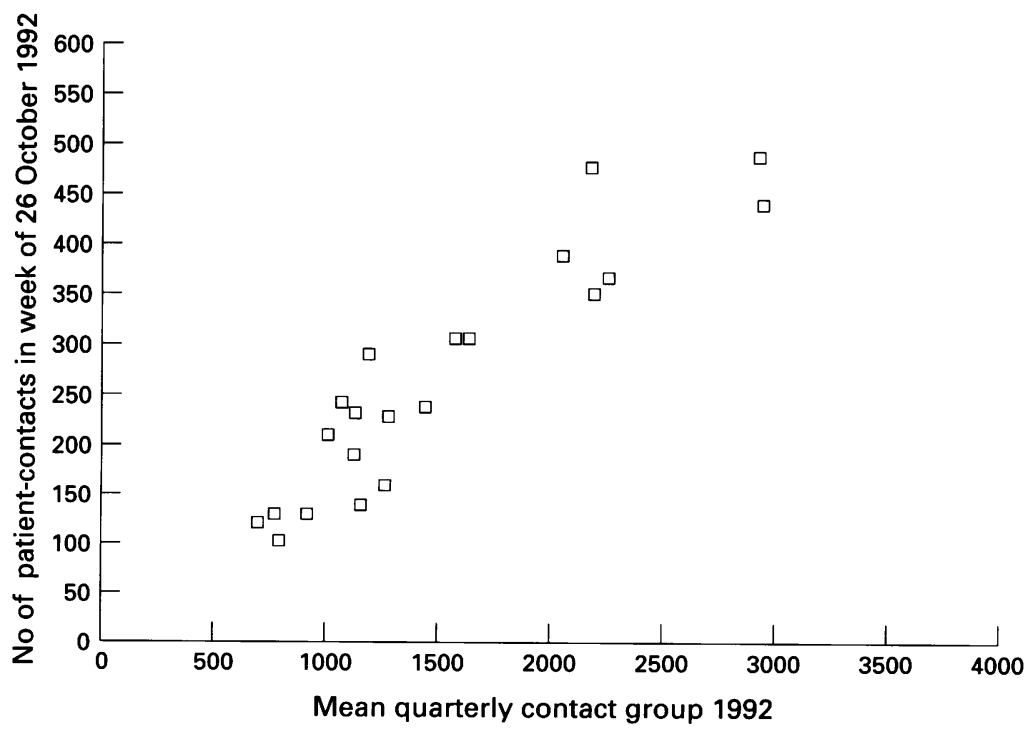

Figure 2 Correlation between the weekly patient contact count in October 1992 and the average quarterly contact group of the sentinel network practices. of privately insured children, estimation of a quarterly contact group is possible by means of the accounting data.

All 26 practices participated in the first weekly contract count. Twenty three practices $(88.5 \%)$ counted all the patient contacts in the other two weeks selected. The same number of practices let us look at their accounting data.

Denominator data were analysed by rank correlation and linear regression methods.

\section{Results}

Paediatric practices had a mean (SD) of 279 $(126 \cdot 8)$ patient contacts in the week of the first count. Although six practices had more than 400 contacts, five saw less than 150 children per week.

The age structure of the children differed significantly between the practices. Figure 1 shows the contact frequency of the five age groups for the average practice and for three practices selected to illustrate the variation in the age structure. Most of the children seen by the sentinel practices were 2 to under 5 years old, $25 \%$ were between 6 months and under 2 years, and another $21 \%$ were between 5 and 10 years old. Children under 6 months and more than 10 years of age were rarely seen. Compared with this average distribution, practice 17 shows a shift to younger children, with more than half of the children younger than 2 years and only $12 \%$ aged 5 years or older. Practice 11 had few contacts with infants under 6 months of age, but the other age groups were almost evenly distributed (between 20 and $27 \%$ of the weekly patient contacts). Practice 21 had an age distribution skewed towards older children with $56 \%$ in school age.

The repeated patient contact count in March 1993 showed higher contact numbers than the first count (mean (SD) 357 (154.9)), but both counts were highly correlated across all practices (Spearman rank correlation coefficient $r_{s}=0 \cdot 86, n=23$ practices).

The rank order of the practices in the different age groups was quite stable, with correlation coefficients between 0.66 for children younger than 6 months and 0.80 for children aged 2 to under 5 years old.

The average quarterly contact group of paediatric practices was estimated as the mean of the four quarterly accounting numbers in 1992 . This mean quarterly contact group ranged from 700 to 3000 treated children per quarter. The distribution was skewed, with most of the practices treating 700 to 1500 children per quarter (median $=1280)$. Rank correlation between these data and the weekly patient contact count in October 1992 yielded an $r_{s}=0.90$, which is even higher than the correlation between the two counts. A scattergram of the correlation is given in figure 2 .

As mentioned above, three of the practices were unable to count total patient contacts in the given week in March 1993. To compensate for this problem of missing data we regressed the number of patient contacts in March 1993 for the other practices on their previous count and on their mean quarterly contact group for 
1992. Multiple $R^{2}$ was 0.89 for $n=21$ practices. The missing contact counts for March 1993 were then estimated by using the regression equation.

\section{Discussion}

To estimate the size of practices in the Hamburg paediatric practices sentinel network and to make time series comparisons possible, total patient contacts per week were counted by the participating practices. The counts showed relevant differences in size and age structure of the practices' patient contacts. We concluded that age specific denominator information should be used and that age standardisation is necessary for purposes of comparison.

Weekly patient contact counts were stable over time, not in terms of the absolute number of contacts but in the rank positions of the practices and in their age structure. One limitation of this study, however, is the fact that the spring and summer season could not be included in the patient census for reasons of feasibility. We do not consider this limitation to be crucial, as differences in practice size could be validated by another data source: quarterly accounting data. Rank correlation was again high. Quarterly contact numbers may be used to estimate single missing data on weekly total patient contacts, but age specific contact information is not available from this source in Germany.

Weekly contact group estimated by counts in a sample of weeks is a useful, stable, and easily available denominator for sentinel practices under the German health care system. It should be interpreted and quoted as a "standardised doctor's week", because computing rates such as, "patient contacts with expiratory wheezing per 100 total patient contacts" would be misleading in that they suggest that both, nominator and denominator, were counted in the same week.

Weekly contact group may be used as a denominator in comparisons over time - that is, to correct for practices that drop out completely or for several (holiday) weeks. It is not, however, an adequate denominator for deriving incidences.

1 Behoerde fuer Arbeit, Gesundheit und Soziales. Gesundheitliche Beeintraechtigungen von Kindern im Umweltzusammenhang. Hamburg; Behoerde fuer Arbeit, Gesundheit und Soziales, 1991.

2 Deffner G, Grieve P, Kloeckner A, Kurme A. Allergische Symptome bei Kindern und Umwelt - ein Pilotprojekt zur Erprobung eines empirischen Zugangsweges. Oeffentliches

3 Kellerhof M. Befundhaeufigkeit in kinderaerztlichen Beobachtungspraxen: Das Pilotprojekt in Hamburg-Harburg. bachtungspraxen: Das Pilotprojekt in Ham

4 Kellerhof M, Kappos AD, Gritz K. Seasonal trends of wheezing related to meteorological and air-quality indicators. Umwelthygiene 1994;(suppl 2):A203. 